\title{
Unbiased segregation of fission yeast chromosome 2 strands to daughter cells
}

\author{
Amar J. S. Klar • Michael J. Bonaduce
}

(C) Springer Science+Business Media Dordrecht (Outside the USA) 2013

\begin{abstract}
The base complementarity feature (Watson and Crick in Nature 171(4356):737-738, 1953) and the rule of semi-conservative mode of DNA replication (Messelson and Stahl in Proc Natl Acad Sci U S A 44:671-682, 1958) dictate that two identical replicas of the parental chromosome are produced during replication. In principle, the inherent strand sequence differences could generate nonequivalent daughter chromosome replicas if one of the two strands were epigenetically imprinted during replication to effect silencing/expression of developmentally important genes. Indeed, inheritance of such a strand- and sitespecific imprint confers developmental asymmetry to fission yeast sister cells by a phenomenon called mating/cell-type switching. Curiously, location of DNA strands with respect to each other at the centromere is fixed, and as a result, their selected segregation to specific sister chromatid copies occurs in eukaryotic cells. The yeast system provides a unique opportunity to determine the significance of such biased strand distribution to sister chromatids. We determined whether the cylindrical-shaped yeast cell distributes
\end{abstract}

Responsible Editors: Helder Maiato and Yves Barral.

A. J. S. Klar $(\bowtie) \cdot$ M. J. Bonaduce

Gene Regulation and Chromosome Biology Laboratory,

National Cancer Institute, Center for National Cancer

Research, National Institutes of Health,

Building 539, Room 154,

Frederick, MD 21702-1201, USA

e-mail: klara@mail.nih.gov the specific chromosomal strand to the same cellular pole in successive cycles of cell division. By observing the pattern of recurrent mating-type switching in progenies of individual cells by microscopic analyses, we found that chromosome 2 strands are distributed by the random mode in successive cell divisions. We also exploited unusual "hotspot" recombination features of this system to investigate whether there is selective segregation of strands such that oldest Watsoncontaining strands co-segregate in the diploid cell at mitosis. Our data suggests that chromosome 2 strands are segregated independently to those of the homologous chromosome.

Keywords Asymmetric cell division mechanism . Somatic DNA strand segregation · Yeast mating-type switching · Cell differentiation

$\begin{array}{ll}\text { Abbreviations } & \\ \text { DNA } & \text { Deoxyribonucleic acid } \\ \mathrm{W} & \text { Watson strand } \\ \mathrm{C} & \text { Crick strand } \\ \text { mat1 } & \text { mating-type gene 1 } \\ \text { mat2-P } & \text { mating-type-donor locus 2 } \\ \text { mat3-M } & \text { mating-type-donor locus 3 } \\ \mathrm{P} & \text { Plus mating type } \\ \mathrm{M} & \text { Minus mating type } \\ \mathrm{Imprint} & \text { A non-mutational epigenetic } \\ & \text { DNA alteration } \\ \mathrm{Mu} & \text { Non-switchable M cell } \\ \mathrm{Ms} & \text { Switchable M cell }\end{array}$


$\mathrm{Pu}$

YEA

PMA

$\mathrm{cM}$

PD

NPD

TT

\section{Introduction}

After two rounds of chromosome replication while pharmacologically blocking centromeric replication, four chromatids originally derived from a single chromosome remain attached to a single centromere. It gives rise to association of four chromatids called diplochromosome. When human blood (Schwarzacher and Schnedl 1966) or marsupial Potorous tridactylis (Walen 1965) kidney cells were labeled with ${ }^{3} \mathrm{H}$-thymidine in the first replication cycle, followed by another cycle without the label, remarkably, the diplochromosome of each metaphase chromosome had only the two outwardly situated chromatids labeled. Thus, DNA strands must hold fixed positions on the centromere in successive rounds of replication and the strands synthesized from the first replication cycle always end up in the "outer" chromatids of the eight-stranded structure. Interestingly, all chromosomes exhibit this behavior. Thus, the location of both "Watson" and "Crick" and older versus firsttime-synthesized strands is fixed at the centromere and the strands are nonrandomly distributed to sister copies in eukaryotic cells (Watson and Crick 1953; Messelson and Stahl 1958). Remarkably, significance of such biased segregation to daughter chromosomes in biology remains unknown. In principle, such an inherent property of the DNA replication process might facilitate the evolution of the selective chromatid segregation phenomenon in eukaryotic cells and such a phenomenon might constitute a mechanism for asymmetric cell division (Klar 2004a). Indeed, chirality of the DNA strands itself causes cellular differentiation by dictating asymmetric cell division in fission yeast Schizosaccharomyces pombe.

A system of heritable switches of the mating-type locus (mat1) in $S$. pombe has been exploited as a model system for several decades to define the mechanisms of directed changes of cell type through asymmetric cell division (for review see Klar 2007). The P (plus) and M (minus) mating/cell types of this organism interchange reciprocally by a transpositionsubstitution recombination process in which a copy of one of the two silenced "donor loci," mat2-P or mat3$M$, is transmitted to the transcription-proficient mat 1 locus (Fig. 1a). Remarkably, switches occur within cell pedigrees by following a highly regular pattern. Specifically, among four granddaughter cells resulting from two successive cell divisions of a "parental cell" (e.g., Mu cell, Fig. 1b), only one granddaughter cell switches in $72 \%$ to $94 \%$ of pedigrees (Miyata and Miyata 1981). Clearly, most cell divisions are developmentally asymmetric and produce a pattern exactly analogous to the stem-cell pattern of cell division (Klar 2007) where one of the daughter cells is equivalent to the parental cell, while the other is advanced in its developmental program. For example, the $\mathrm{Mu}$ (u for unswitchable) cell produces one $\mathrm{Mu}$ "stem cell" and one Ms (s for switchable) cell. Also, the sister of the recently switched cell switches efficiently in subsequent cell divisions as it produces one switched and the other unchanged daughter in over $80 \%$ of cell divisions (Egel and Eie 1987; Klar 1990). That is, the Ms cell mostly produces Ms plus $\mathrm{Pu}$ daughter cells (Fig. 1b). The Pu cell likewise switches back to the $\mathrm{M}$ cell type by following the same patterns of asymmetric cell division. Due to such a high rate of switching, the culture readily becomes a mixture of cells of both types while growing in rich medium. However, cells of opposite mating type mate when starved for nutrients to start meiosis and sporulation developmental program.

The basis for the asymmetric cell division was predicted (Egel 1984) and demonstrated to be due to chromosomal imprinting at the mat1 locus (Klar and Bonaduce 1993), and that too, by a strand-specific mechanism. According to the well-established strandspecific imprint/segregation mechanism (Klar 1987, 1990), a specific strand (say, the "Watson" strand) is imprinted (i.e., a reversible, non-mutational epigenetic DNA alteration) at the mat1 locus during DNA replication (Fig. 1b). Thus, nonequivalent sister chromatids are produced whereby only one of the two mat1 replicas is imprinted, thereby nonequivalent daughter cells result. The daughter cell inheriting the imprinted chromosome from the parental cell acquires switching competence (e.g., Ms in Fig. 1b). Consequently, only one in four granddaughters of an unswitchable cell $(\mathrm{Mu}$ or $\mathrm{Pu}$ ) switches cell type (Fig. 1b). The strand 

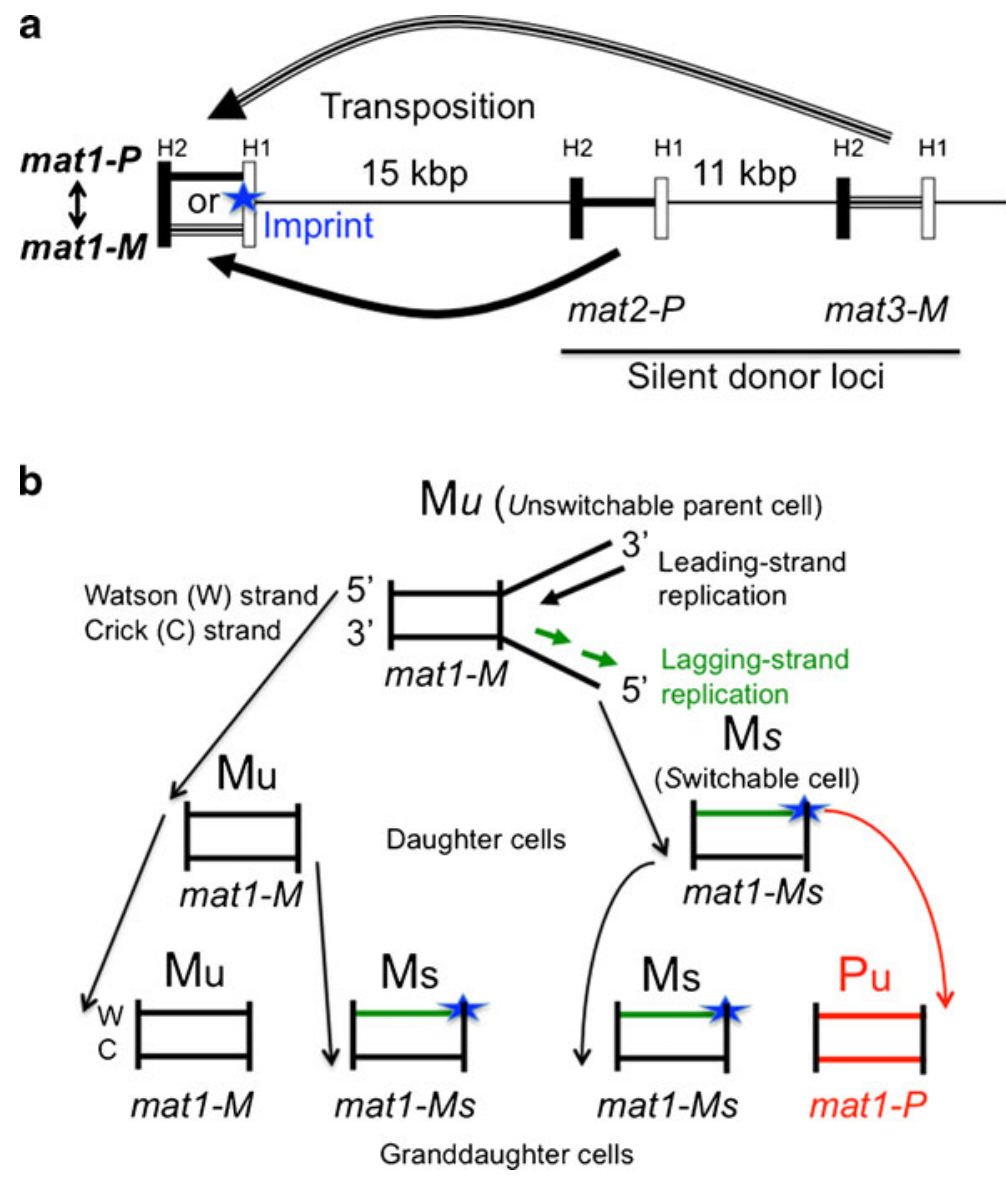

Fig. 1 a The $S$. pombe mat1-switching system (figure modified from Klar 2007). The mat locus spans about $30 \mathrm{kbp}$ in the middle of chromosome 2 . The cell-type determining mat1 locus is switched between two allelic forms by transposing a copy of genetic information, residing in the mat 2 or mat 3 donor locus, through substitution of the resident matlallele. The short $\mathrm{H} 1$ and H2 "homology boxes" present in all cassettes are used to transfer about $1.1 \mathrm{kbp}$ allele-specific sequences copied from donor loci by recombination to produce a switched mat1 allele. The location of the imprint is indicated with a blue-colored star. b The DNA strand-specific imprint/segregation mechanism of

segregation model was first tested genetically by determining the pattern of switching in cell pedigrees of a strain genetically engineered to contain the inverted duplication of the mat 1 locus; its cells produced two cousin switched cells (Klar 1987; Klar 1990), compared with the usual one-in-four granddaughterswitching pattern found in standard strains (Fig. 1b).

The imprint is a site- and strand-specific moiety, either a nick (Klar 1987; Arcangioli 1998; Kaykov and Arcangioli 2004) or a one- or tworibonucleotide moiety inserted in mat1 gene DNA mat1 switching. The two consecutive asymmetric cell divisions in the cell pedigree are due to inheritance of specific parental chromosomal strands by the specific daughter cell such that only one in four granddaughter cells switches. The "Watson" (W) strand is drawn at the top and the "Crick" strand at the bottom. Only the W strand synthesized by the lagging-strand replication complex is imprinted at mat 1 and it is shown by a green-colored line to help follow its distribution in the pedigree. The switched mat $1-P$ allele is presented in red color, whose both strands are synthesized de novo by the mat 1 -switching mechanism. Details are described in the text of the paper

(Klar 1987; Dalgaard and Klar 1999; Vengrova and Dalgaard 2004). Furthermore, the imprint is installed only during the first-time synthesis of the Watson strand and only when replicated by the lagging-strand DNA replication complex (Singh and Klar 1993; Dalgaard and Klar 1999). The imprint creates a DNA fragile site in the chromosome (Beach and Klar 1984; Egel et al. 1984; Nielsen and Egel 1989). DNA replication of the imprinted strand generates a chromatid with a double-stranded DNA break (Beach 1983; Beach 
and Klar 1984) at mat1. The break initiates recombination of mat1 with the donor loci to repair it by gene conversion through the double-strand break repair mechanism (Klar 2007). Thus, this novel DNA strand-based mechanism of cellular differentiation results from asymmetric cell divisions, based on strand-specific imprinting that occurs in one cell cycle and, as a consequence, mat1 switch occurring in one of the daughter cells in the next cell cycle. Notably, chirality of chromosome 2 DNA strands forms the primary basis of both asymmetric cell division and cellular differentiation. Here, we exploit this system genetically to determine the segregation pattern of chromosome 2 strands with respect to cellular polarity in haploid cells. We also define the segregation pattern of replicas of both chromosome 2 homologs in diploid cells.

\section{Materials and methods}

S. pombe strains used in the study

All were diploid stocks: SP10 $\left(h^{90} / m a t 1: 3 M\right.$, his $2 /+$, swi3/+, leu1-32/+, ade6-M210/ade6-M216); SP1146 $\left(h^{90} / h^{90}\right.$, his $2 /+,+/ a d e 1-25,+/ l e u 1-32$, ade6M210/ade6-M216); SP1147 ( $h^{90} /$ mat1-Msmt0, +/his2, ade1-25/+, leu1-32/leu1-32, ade6-M210/ade6-M216); SP1149 $\left(h^{90} / h^{90}\right.$, his2/+, +/ade1-25, leu1-32/leu1-32, ade6-M210/ade6-M216, swi5/swi5); SP1156 (mat1M/mat1-M, $\Delta$ mat2-mat3::LEU2/Dmat2-mat3::LEU2, his2/+, +/ade1-25, +/leu1-32, ade6-M210/ade6M216); SP1157 (mat1-P/mat1-P, Dmat2-mat3:: LEU2/Amat2-mat3::LEU2, his 2/+, +/ade1-25, +/leu1-32, ade6-M210/ade6-M216). The $h^{90}$ genotype reflects strains containing mat1, mat2, and mat3 loci while both mat2 and mat 3 donor loci are deleted in the $\triangle$ mat $2-m a t 3:: L E U 2$ allele (Klar and Miglio 1986).

Culture conditions

Cell growth, construction of genetic stocks, and PMA media used for inducing sporulation were according to the previously described procedures (Moreno et al. 1991). The donor-deleted matl homozygous strains were derived from matl gene conversion events that occurred in cells initially with the mat1-P/mat1-M genotype.
Cell lineage studies

Pedigree analysis to score cells producing asci in cell pedigrees was conducted as described (Klar 1990).

\section{Results}

S. pombe is a unicellular lower eukaryote, usually a haploid organism whose genome is composed of only three chromosomes. Its cylindrical-shaped $(\sim 3-\mu \mathrm{m}$ wide and $\sim 10-\mu \mathrm{m}$ long) cell divides by medial fission to produce nearly equal-sized pairs of daughter/sister cells. The diploid cells we used in our study were correspondingly bigger than haploid cells. The single cell elongates and divides while growing on the surface of solid medium, and because cells are immotile, daughter cells maintain their relative orientation with respect to each other during their own division. For conducting our experiments, we exploited a combination of several unique properties of the system; invariant rod-shaped cell structure, immotile cells, onedimensional growth at the cell's tips causing it to become longer before division, cell division by fission (hence it is called fission yeast) by medial septum formation, a high rate of recurrent mating-type

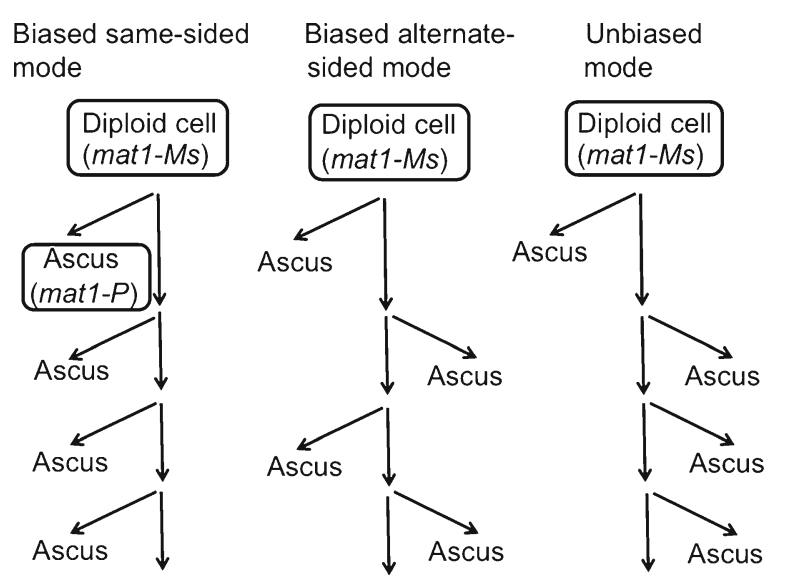

Fig. 2 Diagrams of three hypothetic modes of strand distribution to specific poles of the parental cell. The pedigree is conducted with diploid SP10 $\left(h^{90} /\right.$ mat $\left.1: 3 M\right)$ cells permitting us to score sporulation ability of individual cells. After mat $1-M$ to mat1-P switch had occurred in the chromosome containing the mat $1-M$ imprinted locus, equivalent to the Ms allele in Fig. 1b, the recently switched cell with the mat1-P allele would form an ascus. Segregation mode of a specific strand of one of the chromosome 2 copies was identified by microscopically observing ascus formation by the progenies of single cells 
switching, and, most importantly, the inheritance of a specific chromosome 2 strand causes the genetically heritable mat1 switch. Moreover, the recently switched cell can be identified at the single-cell level through microscopic observations. These features were exploited here to determine the mode of strand distribution in successive cycles of cell division of diploid cells.

Possible modes of chromosome 2 strand segregation in successive mitoses

The diplochromosome studies quoted above have suggested that the position of the Watson and Crick and old versus newly synthesized strands on the centromere is fixed and that they are segregated in a biased pattern to daughter chromosomes in successive cycles of chromosome replication. Should the centromere maintain a precise orientation in the cell with respect to cellular morphology, it is possible that chromosome 2 strands may be distributed in a regulated pattern with respect to cellular poles during successive cell divisions. We can imagine two regulated biased and one unbiased modes of strand distribution to daughter cells by the cylindrical-shaped yeast cell (Fig. 2): (1) the specific strand conferring mating-type switching competence would be distributed to the same side of the sister-sister cells pair by the biasedsame-sided mode or (2) the specific strands would be distributed strictly in an alternate fashion in successive cell divisions in the biased-alternate mode. In the unbiased mode, non-patterned distribution would occur. The inheritance pattern was determined by recording the spatial relationship of switched cells produced by consecutive cell divisions of individual cells, as described below.

The system exploited to determine mode of strand segregation in successive mitoses of yeast cells

The diploid strain SP10 (mat $1-M$ or P/mat 1:3-M genetic constitution; see "Materials and methods" for description of the complete genotype) was previously employed for determining the pattern of switching by pedigree analysis (Klar 1990). It contains wild-type mat1 in one chromosome, whose pattern of switching we wished to determine. The other homolog contains a mat 1:3- $M$ fusion cassette due to a genetic rearrangement of mat 1 and mat 3 genes causing a deletion of about $28.0 \mathrm{kbp}$ intervening sequences (Beach et al. 1982).
This deletion removes the sequences essential for imprinting (Arcangioli and Klar 1991); consequently, the mat 1:3-M hybrid cassette is incapable of switching, but it is capable of providing sporulation functions of the mat $1-M$ allele. The mat $1-M /$ mat $1: 3-M$ cells are unable to undergo meiosis and sporulation as both alleles contain $M$-information, but the mat1-P/mat1$M$ heterozygous constitution is essential for inducing sporulation (Kelly et al. 1988). However, once mat1$M$ switch had generated the mat $1-P$ allele, the cell with the resulting mat1-P/mat1:3-M constitution becomes competent to undergo sporulation under nutritional starvation conditions. The switched cells sporulate, while the unchanged cells continue to divide under the specific growth/sporulation conditions we employed. With this procedure, an ascus formed by a cell identified a recently switched cell (Fig. 2). Previous such pedigree studies (Egel and Eie 1987; Klar 1990) did not determine the pattern of strand segregation since the data on the relative location of switched versus unswitched cells within the sister-sister pairs in consecutive mitoses were not collected. In those studies, the sister cells were separated from each other by micromanipulation, and this procedure disrupts the relative orientation of sister cells. In our study, we particularly determined the spatial relationships of switched (i.e., sporulated) cells by investigating successive rounds of cell divisions.

Once a cell has initiated meiosis, it produces an ascus containing four rounded haploid products, called ascospores. Notably, ascospores do not grow on the sporulation medium that we employed for the pedigree analysis. Mitotic and meiotic cells were morphologically distinguished from each other by microscopic observations of cells growing and sporulating on the surface of solid medium. This procedure allowed us to observe the pattern of mating-type switching of daughter chromosomes in multiple cycles of cell growth. Specifically, we monitored whether an ascus produced by a dividing cell is always placed on the same side of the rodshaped cell or always on the opposite side, when compared to the one from the previous division (Fig. 2), or whether the asci are randomly placed. Under these starvation conditions, the cells elongated $\sim 2$-fold when compared with the length of cells growing in rich medium, a feature making our analysis feasible. 
With micromanipulator, we placed individual cells on the surface of solid PMA (sporulation) medium. We started our analysis of pairs of sister cells, one of which had produced an ascus. There, the sporulated cell had switched to mat1-P allele since it formed an ascus, and the unswitched sister cell must have inherited Ms imprinted mat 1 allele in over $80 \%$ of cases (Fig. 1b). Following this procedure, individual Ms cells were identified and subjected to further pedigree analysis. The asci were removed by micromanipulator after recording their relative orientation with respect to the unswitched sib cell and its progeny. By microscopically observing the growth of cells in otherwise undisturbed pedigrees, we recorded whether the ascus produced in a given cell division was placed on the same side or on the opposite side, relative to the axis of division, as compared to the relative location of an ascus in the prior division. In total, we noted 28 cases in which the subsequent switched cell was located on the same side to that exhibited by the switched cell in a prior generation and 25 cases were opposite-sided. These results rule out both biased patterns and demonstrate the unbiased mode of DNA strand distribution with respect to the polarity of the cell (Fig. 2).

The mat 1 hotspot produces a recombinant class homozygous for markers located centromere-distal to the crossover point

The mat1 imprint, employed by yeast for mat 1 switching (Fig. 1a), is also a "hotspot" of mitotic recombination in diploid cells. Angehrn and Gutz (Angehrn and Gutz 1968; Gutz et al. 1974) first reported a very high rate of mitotic recombination in $\mathrm{S} / \mathrm{G} 2$ phase at mat1. Cells growing in rich medium have essentially non-existing G1phase, very short S phase length, and most of the cell-cycle length consists of the G2 phase (Gutz et al. 1974). About $2 \%$ mitoses of $+/$ his 2 cells produce recombinant cells homozygous for the mat1-distal his 2 marker and for all other distal markers on the chromosome 2 arm (Angehrn and Gutz
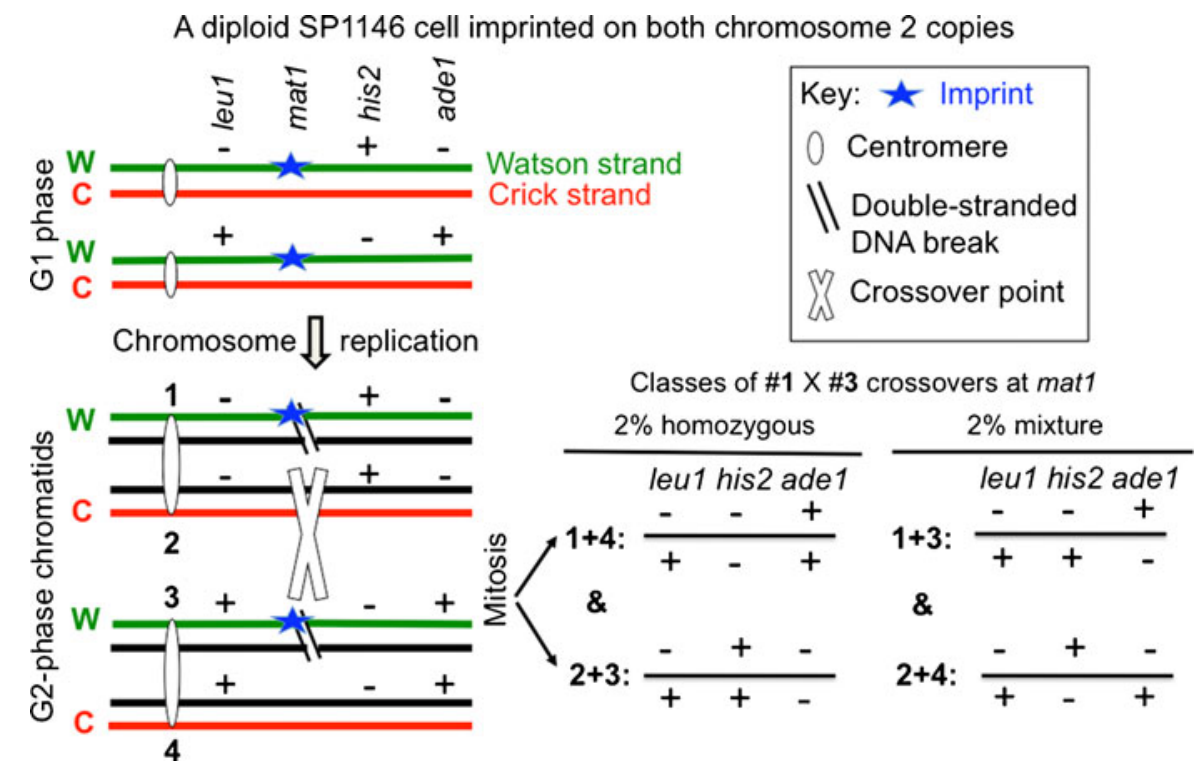

Fig. 3 The chromosome 2 arms swapping hypothesis. It is advanced to explain the recombination mechanism of the mat 1 hotspot. The location of genetic markers is not drawn to scale. The meiotic genetic distance between leul and mat 1 markers is $10 \mathrm{cM}$, mat 1 and his 2 is $2.0 \mathrm{cM}$, and between mat 1 and ade 1 is $>50 \mathrm{cM}$. According to our arms-swapping hypothesis, recombination occurs only between non-sister chromatid \#1 with \#3 and only when they simultaneously have DNA breaks (lcr) in the S/ G2 phase of the cell. To depict specific strand distribution, template Watson $(W)$ strands are colored green and Crick $(C)$ ones are colored red. Strands synthesized in the present replication cycle are depicted in black color. The symbol $X$ represents the crossover point at matl. The numbers 1 to 4 represent chromatid number assigned to their centromeres. Two classes of recombinants concerning genetic markers flanking mat1 are shown. The $1+4$ and $2+3$ chromatid segregation, where each daughter cell inherits a single recombined chromatid, causes homozygosis of all markers distal to the crossover point. When both recombined chromatids $1+3$ cosegregate to one of the daughter cells, it remains heterozygous for all the markers, but notably, coupling of flanking markers changes due to recombination 


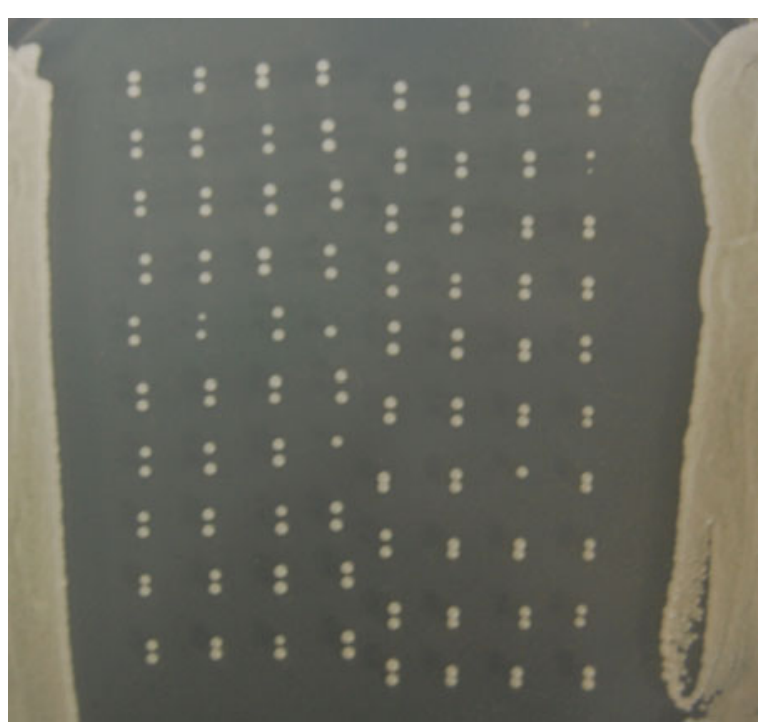

Fig. 4 Homozygosis experiment. The procedure tests phenotype of colonies seeded with sister cell pairs after their growth on rich (YEA) medium. The adjacent colonies in the picture represent those seeded with sister cell pairs. These colonies were scored for his 2 and adel markers by replica plating them to appropriate drop-out media

1968; Egel 1981). The his2 marker is closely linked to mat 1 by a meiotic distance of $2.0 \mathrm{cM}$ representing about $40.0 \mathrm{kbp}$ length. The homozygosis occurs in cases when each daughter cell inherits one of the two recombined chromatids (Fig. 3). Homozygous events were quantified by replica plating colonies of sistersister cell pairs to the appropriate medium to uncover recessive auxotrophic mat1-distal markers, for example, the his 2 and ade1 markers (Fig. 3).

We constructed the SP1146 diploid strain for our study. The alleles of relevant three markers, leu1, his2, and adel, were arranged in the order as presented in Fig. 3. For hotspot recombination experiments, sister-sister cell pairs growing on the surface of solid YEA medium were separated and planted side-by-side by micromanipulation
(Fig. 4). After growth for 3 days, the resulting colonies were tested for the his 2 and adel markers by replica plating them to appropriate media. Homozygosis uncovered recessive markers; one cell produced his2/his2, and its sister produced adel/ade1 colonies. Following mitotic recombination at mat1, homozygosis of originally heterozygous markers occurred in $\sim 2 \%$ of cell divisions (Table 1). This finding confirms the results of earlier studies (Angehrn and Gutz 1968; Egel 1981), and it extends the analysis to the ade 1 markers located $>50 \mathrm{cM}$ distal to the mat1 locus.

The hotspot recombination fails when one of the chromosome partners is not imprinted

It was discovered previously that mat1 hotspot recombination rate (i.e., homozygosis) is reduced roughly in proportion to the reduced matl-switching rate of mat1-cis-acting mutation carried in one of the homologs, although the other homolog carried the wild-type mat1 allele (Angehrn and Gutz 1968; Egel 1981). This finding remains an unexplained feature of the hotspot recombination. It is generally thought that recombination efficiency is limited by the frequency of the recombination initiating event, such as the doublestranded chromosomal break (Strathern et al. 1982; Szostak et al. 1983) and that once initiation has occurred in one of the chromosomes/chromatids, its recombination and DNA repair ensues efficiently by interactions with the sister chromatid and/or with the homolog. Why does the wild-type mat1 locus present in one of the homologs not undergo efficient recombination in the diploid cell when the other mat 1 allele is compromised in switching ability? We wished to explore the reason for this highly unusual property of the hotspot recombination mechanism.

To further explore the hotspot recombination process, we analyzed strain SP1147 $\left(h^{90} /\right.$ mat $\left.1-M s m t 0\right)$.

Table 1 Hotspot recombination

\begin{tabular}{lllllr}
\hline Strain & mat1 & mat2 and mat3 & swi5 & Homozygosed sisters & \% homozygosis \\
\hline SP1146 & $h^{90} / h^{90}$ & $+/+$ & $+/+$ & $14 / 660$ & 2.1 \\
SP1147 & $h^{90 / m a t 1 M s m t 0}$ & $+/+$ & $+/+$ & $0 / 1,068$ & $<0.1$ \\
SP1149 & $h^{90} / h^{90}$ & $+/+$ & $-/-$ & $13 / 494$ & 2.6 \\
SP1156 & mat1-M/mat1-M & $\Delta / \Delta$ & $+/+$ & $7 / 400$ & 1.75 \\
SP1157 & mat1-P/mat1-P & $\Delta / \Delta$ & $+/+$ & $7 / 358$ & 1.95 \\
\hline
\end{tabular}


The smt0 mutation is a 254-bp deletion of mat1-cisacting sequences that are essential for imprinting (Styrkarsdottir et al. 1993). Thus, only the $h^{90}$ chromosome can be imprinted in this diploid strain. Indeed, the SP1147 strain did not undergo homozygosis at all (Table 1). To account for this observation and for those quoted above (Angehrn and Gutz 1968; Egel 1981), we propose that recombination occurs only in those cells that simultaneously contain two non-sister chromatids both with the DSB (Fig. 3). Simultaneous imprinting of both homologs would occur in a minority of diploid cells. We also propose that transiently broken chromatid arms in S/G2 phase are simply swapped between homologs, much like the topoisomerase activity that exchanges two DNA partners without involving the DNA synthesis machinery (Fig. 3).

The recombination-deficient swi5/swi5 mutant is proficient in hotspot recombination

To address this specific chromatids" "arms swapping" hypothesis, we tested the hotspot activity in the recombination-deficient swi5/swi5 mutant. The swi5 mutant is defective for mat1 switching in mitosis (Egel et al. 1984). Consistent with our hypothesis, the swi5/swi5 mutant (SP1149) strain exhibited homozygosis at the same rate as that found in wild-type SP1146 cells (Table 1).

Donor-deleted strains exhibit hotspot activity

One complication that we faced in our hotspot studies is that mat1-mat 2 and mat1-mat3 loci fusions, that delete intervening sequences, spontaneously arise in cultures at appreciable frequencies as a byproduct of the mat1-switching phenomenon (Beach and Klar 1984). Due to the deletion in one of the homologs, hotspot activity is lost in such strains. To prevent generation of such rearrangements during our analysis, we next employed strains that were deleted for both donor loci (Klar and Miglio 1986). Indeed, we found that both mat1-P/mat1-P (SP1156) and mat1-M/mat1-M (SP1157) donor-deleted strains undergo homozygosis at the usual rate (Table 1) initially reported for $h^{90}$ strains (Angehrn and Gutz 1968; Egel 1981). Our results show that donor-deleted mutations do not influence the hotspot activity.
The mat 1 hotspot produces a recombinant class with altered coupling of markers flanking the crossover point

As a measure of overall mitotic recombination frequency at the hotspot, it was presumed that, at a similar frequency of $2 \%$ causing homozygosis noted above, both recombined chromatids are segregated to the same daughter cell $(1+3$ chromatid segregation class in Fig. 3) (Egel 1981). Because they would maintain heterozygosity of all markers, like the cells of the parental culture, their identification is problematic. As a consequence, this class of recombinants has not been investigated. However, this class can be identified because it should alter the coupling of mat1 locus flanking leu1 and his2 (plus ade1) alleles when compared to the arrangement of the parental stock (Fig. 3). We next addressed the existence of this class in SP1146 culture by tetrad analysis.

Because homozygosis occurs in $2 \%$ of cells at each cell division, the stock originally heterozygous for his 2 and ade1 markers (Fig. 3) readily becomes a mixture of his2/his 2 and ade1/ade1 cells. We constructed fresh SP1146 hybrid colonies by mating the appropriate haploid strains, grew them on synthetic minimal medium for $48 \mathrm{~h}$ to prohibit growth of his2/his 2 and adel/adel homozygosed cells produced in the culture, and then transferred them to PMA medium (supplemented with leucine, histidine, adenine, and uracil) for meiosis and sporulation induction. We note that this experiment was performed with cells derived from individual colonies. With his regime, we selected against those cells that had homozygosed during growth of the colony on minimal medium, and equally importantly, we reduced the number of intervening cell divisions of the hybrid to a minimum before their meiosis and sporulation was induced. We performed tetrad analysis of thus produced asci of cells derived from three independently constructed SP1146 hybrids (Fig. 5).

Because leul and his2 markers are situated only $10 \mathrm{cM}$ (meiotic distance) apart, the frequency of nonparental ditype (NPD) tetrads in our cross should be very rare because their generation requires more than one crossover event to occur between the markers in a single meiosis. In contrast, all three hybrids (\#3 to 5) we analyzed contained nearly one quarter of cells that generated the NPD class of tetrads. As a consequence, the apparent map distance of about $10 \mathrm{cM}$ (meiotic 


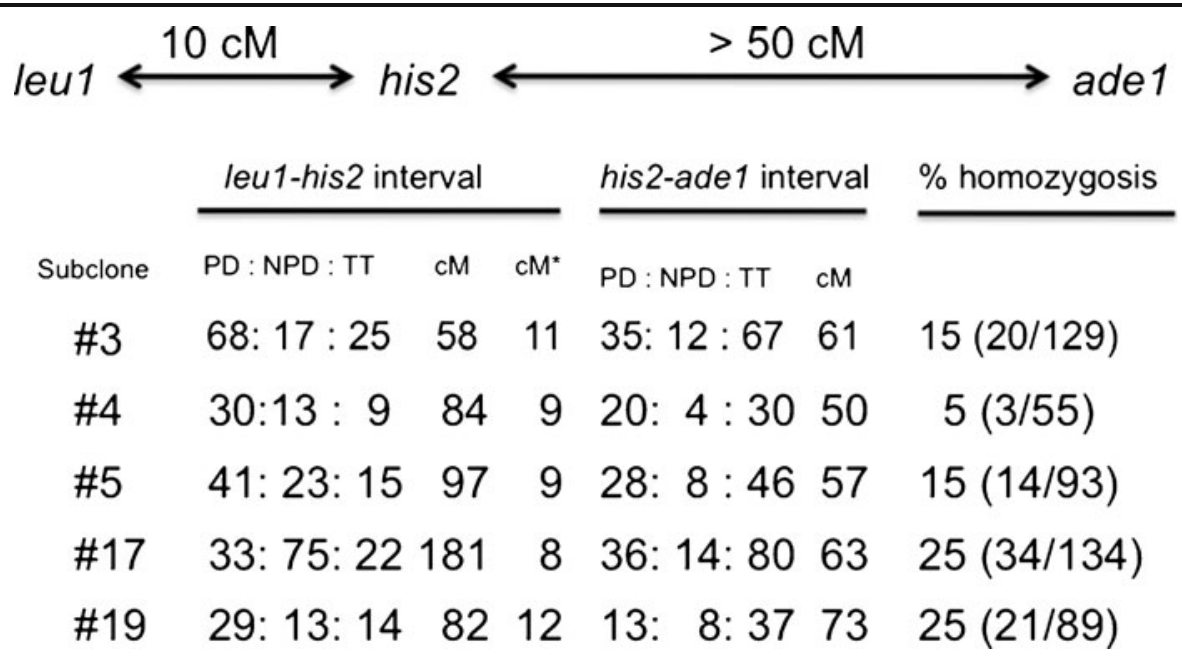

Fig. 5 Classes of recombination due to hotspot. Homozygosis frequency and markers map distance in strain SP1146 subclones is reported. The values of meiotic genetic distance from the literature of markers of conventional strains without hotspot recombination are indicated at the top. Results of tetrad analysis

are tabulated. $P D$ parental ditype, NPD non-parental ditype, $T T$ tetra-type tetrads, $c M$ the apparent meiotic map distance calculated in centimorgans, $c M^{*}$ adjusted meiotic map distance calculated by deducting the contribution of hotspot recombination

map distance) between these markers had expanded between three and fivefold. Significantly, the proportion of NPD tetrads of the leu1-his2 interval was several folds higher than that found for the much larger his2-adel interval where conventional meiotic recombination had occurred (Fig. 5). The preponderance of NPD class of tetrads was predicted to result from cells in which change in coupling of leul and his 2 alleles have occurred, with respect to the parental combination, during mitosis due to hotspot recombination. Clearly, NPD class represented tetrads of cells that inherited $1+3$ chromatids following mitotic hotspot recombination (Fig. 3). Moreover, tetrads of cells homozygosed for his 2 and adel alleles were produced by each hybrid. This was expected because homozygosed cells produced on sporulation medium were not selected against.

To quantify all recombination classes diagrammed in Fig. 3, we next determined the genetic composition of unselected cells from an individual colony. For this purpose, subclone \#17 of hybrid SP1146 was processed differently from those cultures discussed above. In this case, we did not select against homozygosed cells. Specifically, a single colony grown on rich YEA medium-containing plate was transferred to a plate containing PMA (supplemented with leucine, histidine, adenine, and uracil) medium by replica plating. Their asci were subjected to tetrad

analysis. Interestingly, this colony produced NPD tetrads (Fig. 5) more frequently than those exhibiting parental ditype (PD). Normally, it is impossible to obtain a NPD $>$ PD result for linked markers. We explain this result by presuming that the cell from which colony \#17 originated had already changed the coupling of mat1 flanking markers due to the hotspot activity. Thus, PD class here represented cells in which both recombined chromatids $(1+3$ class, Fig. 3$)$ were delivered to the same daughter cell following recombination at the hotspot in mitosis. Notably, 34 tetrads represented cells that had homozygosed and 33 cells produced PD tetrads reflecting cells where coupling of markers flanking matl has changed (Fig. 5). Similarly, colony \#19 results also showed increased map distance in the leu1-his2 interval. In all five cultures analyzed, the number of cells that had homozygosed was similar to the number of minority class of cells that produced NPD (but PD in colony \#17) (Fig. 5). These observed similar frequencies support the notion that recombined chromatids are segregated to daughter cells in an unbiased manner. We propose that the increased map distance is an artifact introduced by the hotspot recombination occurring in mitosis but not in meiosis. Indeed, when we adjust for the contribution of the hotspot recombination to the observed map distance, by counting PD- and NPD-producing cells having not been recombined in meiosis, the leu1- 
his 2 interval comprised between 8 and $12 \mathrm{cM}\left(\mathrm{cM}^{*}\right.$ row in Fig. 5). Notably, this distance is similar to that of about $10.0 \mathrm{cM}$ found in standard strains (Fig. 5, top). Collectively, our results from the analyses of five different hybrids suggest that both classes of recombinant between chromosome 2 homologs are produced due to the hotspot recombination (Fig. 3). We obtained no evidence supporting the idea that there is a biased distribution of recombined chromatids to daughter cells. Therefore, we surmise that recombined chromatids are distributed to both daughter cells versus to the single daughter cell with a similar frequency.

\section{Discussion}

We can make several conclusions from recombination studies at the mat1 hotspot: (1) recombination occurs in $\mathrm{S} / \mathrm{G} 2$ phase, similar to the phase in which mat1 switching usually occurs (Fig. 1b); (2) recombination occurs only in S/G2 phase in meiosis as well (Klar and Miglio 1986); (3) recombination occurs between nonsister chromatids at an appreciable rate; (4) recombination occurs only when two non-sister chromatids simultaneously contain DSB in the same cell; and (5) recombination occurs only in specific non-sister chromatids (those inheriting previously imprinted Watson strands; Fig. 3). Our results support the arms swapping hypothesis (Fig. 3) advanced to explain the mechanism of mitotic recombination at the mat1 hotspot. Assuming that $40 \%$ of chromosomes are imprinted at mat $1,16 \%(40 \% \times 40 \%)$ of diploid cells in G2 phase would contain simultaneously cleaved chromatids. We imagine that broken ends of chromatids are equally likely stitched together, preserving the original genetic configuration, similar to the frequency of those events where non-sister chromatids had been swapped to cause recombination. It is possible that a recombination intermediate is formed and it can isomerize such that only one half of resolution events result in crossovers. Thus, at a maximum only $8 \%(16 \% / 2)$ cell divisions could undergo crossover events. Assuming that $4 \%$ cells exhibit recombination (Fig. 3), thus the hotspot recombination is an efficient process. Future research should address molecular details of this unusual recombination mechanism. Here, we analyzed events of recombination occurring between non-sister chromatids; we note that yeast can also repair the chromatid break through interactions with the sister chromatid (Klar and Miglio 1986). Most uniquely, special features of recombination occurring only in S/G2 phase and only between specific non-sister chromatids have allowed us to identify the mode of strand segregation in mitosis. Because both $1+4: 2+3$ and $1+3: 2+4$ chromatid segregation types are found to occur equally frequently (Figs. 3 and 5), we suggest that the chromosome 2 strands of one homolog are segregated randomly and independently with respect to those of the second homolog during cell division.

We also exploited several unique features of the mat1 switching system to determine the segregation pattern of strands of a single chromosome by genetic studies with fission yeast. Many studies have been conducted to determine the pattern of strand segregation of the whole genome in several organisms. Cairns (1975) first hypothesized the requirement of nonrandom distribution of DNA strands of all chromosomes in rapidly proliferating epithelial cells for minimizing accumulation of mutations produced by DNA replication. It was proposed that stem cells might always inherit template-containing DNA strands allowing them to escape cancer-causing mutations. In support of the genome-wide directedsegregation mechanism, nonrandom segregation of sister chromatids was observed in some studies that followed the distribution of bromo-deoxyuridine- or radio-labeled chromosomes (Lark et al. 1966; Rosenberger and Kessel 1968; Potten et al. 1978; Merok et al. 2002; Smith 2005), while the random pattern was found in other studies (Ito and McGhee 1987; Yadlapalli et al. 2011). Another interesting case of selective segregation of chromatids of only the paternal set of chromosomes in the apical cells of shoots and roots of the water fern Marsilea vestita has been reported (Tourte et al. 1980).

It is noteworthy that results of studies testing genome-wide segregation of labeled strands are informative only if the entire genome undergoes nonrandom distribution. If nonrandom segregation occurs only in a subset of chromosomes, the labeling procedure would not provide interpretable information. Also, questions arise whether nonrandom distribution is always an inherent aspect of the DNA replication machinery and, should it occur, what function does it serve. A purpose for evolving the selective strand-segregation mechanism has been suggested whereby DNA replication may modulate transcriptional 
potential of developmentally important genes in a strand-specific fashion, perhaps by differential organization of chromatin structure of sister chromatids of both homologs. Accordingly, chromosome-specific nonrandom segregation of thus differentiated sister chromatids might result in production of nonequivalent daughter cells (Klar 2004b). In contrast to studies testing co-segregation of the entire genome, our study is unique in at least two aspects. We were able to follow strand segregation of a single chromosome and uniquely by assessing the cellular differentiation of individual cells in mitosis.

We discovered a random pattern of chromosome 2 strand distribution to daughter cells in successive cell divisions with respect to parent yeast cell's poles. In this simple unicellular organism - exhibiting only two kinds of cell type - the particular daughter cell exhibits the program based on the developmental potential carried by the specific chromosome inherited from the parental cell. Because yeast is a haploid unicellular organism, no obvious advantage can be imagined for the existence of a patterned distribution process. Due to the special experimentally amenable features of yeast discussed above, a strand-specific mechanism for cellular differentiation was only tested and established in the $S$. pombe system. Unlike yeast, sister cells during asymmetric cell division in higher eukaryotes are often nonrandomly placed with respect to adjoining cells (Horvitz and Herskowitz 1992). Epigenetically mediated states of gene expression can be inherited chromosomally through multiple cell divisions (Grewal and Klar 1996; Thon and Friis 1997), and the opportunity for altering the epigenetic state during DNA replication exists (Zhang et al. 2000). Therefore, the possibility for biologically relevant biased segregation of chromatids from both homologs can be imagined. We suggest that strandspecific imprinting along with nonrandom segregation mechanisms may have evolved as one of the avenues to produce multiple arrays of cell types in higher eukaryotes (Klar 2004b). In mouse embryonic stem cells, the data indicates that parental chromosome 7 Watson strands always co-segregate to the same daughter cell leaving Crick strands to go to the sister cell (Armakolas and Klar 2006; Armakolas and Klar 2007). Such biased chromatid segregation is proposed to be a mechanism for development in higher organisms (Klar 2008; Armakolas et al. 2010; Sauer and Klar 2012). Because Cre/Lox-induced chromosome 7 mitotic recombinants are always of homozygous type for markers distal to the crossover point, similar to the homozygosis outcome in yeast (Fig. 3), we previously proposed that recombination only occurs in G2 phase with selected non-sister chromatids and therefore deduced that selective chromatid segregation occurs in mouse embryonic stem cells. It was not possible to directly determine the basis of recombination restriction to G2 phase and of selected chromatid recombination in mouse cells. In the $S$. pombe cells, however, we conclude here that recombination occurs only in S/G2 phase in selected non-sister chromatids due to strand-specific imprinting but chromatids are segregated to daughter cells through the unbiased mode (Fig. 3).

It is essential to develop techniques to determine segregation patterns of individual chromosomes of the genome. By labeling the budding yeast Saccharomyces cerevisiae genome for one cell cycle by incorporating 5bromo-deoxyuridine nucleotide analog, then analyzing chromosomes of mother cells separated from those of daughter cells, it was recently reported that there is no mother-daughter bias in segregation of specific strands of any chromosome (Keyes et al. 2102). In contrast, it was recently reported that preexisting histones are selectively retained in Drosophila male germline stem cells, while newly synthesized histones are enriched in the differentiating daughter cell (Tran et al. 2012). Thus, an active area of research is aimed at determining the strand segregation pattern and exploring their significance in biology at large. Thus far, $S$. pombe has been the only system examined and shown to accomplish cellular differentiation strictly based on the chirality of the DNA molecule (Klar 1987, 1990). We recently reported that such a system also operates in Schizosaccharomyces japonicus, an organism that shares only about $30 \%$ DNA sequence identity with genes of S. pombe (Yu et al. 2013). These two evolutionarily highly diverged organisms demonstrate remarkable conservation of a DNA strand-based mechanism of asymmetric cell division. It remains a major challenge to design strategies for determining whether such a mechanism for cellular differentiation has evolved in multicellular organisms.

Acknowledgments We thank our laboratory colleagues Sharon Moore, Stephan Sauer, and Ken Ishikawa for discussions and for critical reading of the manuscript. The Intramural Research Program of the National Cancer Institute, National Institutes of Health, supports our research. 
Author contributions A.K. designed the project, performed experiments, analyzed data, and wrote the manuscript, and M.B. provided technical support for this work.

Conflict of interest The authors declare that they have no conflict of interest.

\section{References}

Angehrn P, Gutz H (1968) Influence of the mating type on mitotic crossing-over in Schizosaccharomyces pombe. Genetics 60:158. Abstract

Arcangioli B (1998) A site- and strand-specific DNA break confers asymmetric switching potential in fission yeast. EMBO J 17(15):4503-4510

Arcangioli B, Klar AJS (1991) A novel switch-activating site (SAS1) and its cognate binding factor (SAP1) required for efficient matl switching in Schizosaccharomyces pombe. EMBO J 10:3025-3032

Armakolas A, Klar AJS (2006) Cell type regulates selective segregation of mouse chromosome 7 DNA strands in mitosis. Science 311:1146-1149

Armakolas A, Klar AJS (2007) Left-right dynein motor implicated in selective chromatid segregation in mouse cells. Science 315:100-101

Armakolas A, Koutsilieris M, Klar AJS (2010) Discovery of the mitotic selective chromatid segregation phenomenon and its implications for vertebrate development. Curr Opin Cell Biol 22(1):81-87

Beach D, Nurse P, Egel R (1982) Molecular rearrangement of mating-type genes in fission yeast. Nature 296:682-683

Beach DH (1983) Cell type switching by DNA transposition in fission yeast. Nature 305:682-688

Beach DH, Klar AJS (1984) Rearrangements of the transposable mating-type cassettes of fission yeast. EMBO J 3:603-610

Cairns J (1975) Mutation selection and the natural history of cancer. Nature 255:197-200

Dalgaard JZ, Klar AJS (1999) Orientation of DNA replication establishes mating-type switching pattern in $S$. pombe. Nature 400:181-184

Egel R (1981) Mating-type switching and mitotic crossing-over at the mating type locus in fission yeast. Cold Spring Harbor Symp Quant Biol 45:1003-1007

Egel R (1984) The pedigree pattern of mating-type switching in Schizosaccharomyces pombe. Curr Genet 8:205-210

Egel R, Beach DH, Klar AJS (1984) Genes required for initiation and resolution steps of mating-type switching in fission yeast. Proc Natl Acad Sci U S A 81:3481-3485

Egel R, Eie E (1987) Cell lineage asymmetry for Schizosaccharomyces pombe: unilateral transmission of a high-frequency state of mating-type switching in diploid pedigrees. Curr Genet 3:5-12

Grewal SIS, Klar AJS (1996) Chromosomal inheritance of epigenetic states in fission yeast during mitosis and meiosis. Cell 86(1):95-101

Gutz H, Heslot H, Leupold U, Loprieno N (1974) In: King RC (ed) Schizosaccharomyces pombe. Plenum, New York, pp 395-446
Horvitz HR, Herskowitz I (1992) Mechanisms of asymmetric cell division: two Bs or not two Bs, that is the question. Cell 68(2):237-255

Ito K, McGhee JD (1987) Parental DNA strands segregate randomly during embryonic development of Caenorhabditis elegans. Cell 49(3):329-336

Kaykov A, Arcangioli B (2004) A programmed strandspecific and modified nick in $S$. pombe constitutes a novel type of chromosomal imprint. Curr Biol 14(21):1924-1928

Kelly M, Burke J, Smith M, Klar A, Beach D (1988) Four mating-type genes control sexual differentiation in the fission yeast. EMBO J 7(5):1537-1547

Keyes BE, Sykes KD, Remington CE, Burke DJ (2102) Sister chromatids segregate at mitosis without mother-daughter bias in Saccharomyces cerevisiae. Genetics 192:15531557

Klar AJS, Miglio LM (1986) Initiation of meiotic recombination by double-strand DNA breaks in S. pombe. Cell 46(5):725-731

Klar AJS (1987) Differentiated parental DNA strands confer developmental asymmetry on daughter cells in fission yeast. Nature 326(6112):466-470

Klar AJS (1990) The developmental fate of fission yeast cells is determined by the pattern of inheritance of parental and grandparental DNA strands. EMBO J 9(5):1407-1415

Klar AJS (2004a) A genetic mechanism implicates chromosome 11 in schizophrenia and bipolar diseases. Genetics 167(4):1833-1840

Klar AJS (2004b) An epigenetic hypothesis for human brain laterality, handedness and psychosis development. Cold Spring Harb Symp Quant Biol 69:499-406

Klar AJS (2007) Lessons learned from studies of fission yeast matingtype switching and silencing. Annu Rev Genet 41:213-236

Klar AJS (2008) Support for the selective chromatid segregation hypothesis advanced for the mechanism of leftright body axis development in mice. Breast Dis 29:47-56

Klar AJS, Bonaduce MJ (1993) The mechanism of fission yeast mating-type interconversion: evidence for two types of epigenetically inherited chromosomal imprinted events. Cold Spring Harb Symp Quant Biol 58:457-465

Lark KG, Consigli RA, Minocha HC (1966) Segregation of sister chromatids in mammalian cells. Science 154(753):12021205

Merok JR, Lansita JA, Tunstead JR, Sherley JL (2002) Cosegregation of chromosomes containing immortal DNA strands in cells that cycle with asymmetric stem cell kinetics. Cancer Res 62(23):6791-6795

Messelson M, Stahl FW (1958) The replication of DNA in Escherichia coli. Proc Natl Acad Sci U S A 44:671-682

Miyata H, Miyata M (1981) Mode of conjugation in homothallic cells of Schizosaccharomyces pombe. J Gen Appl Microbiol 27:365-371

Moreno S, Klar A, Nurse P (1991) Molecular genetic analysis of fission yeast Schizosaccharomyces pombe. Methods Enzymol 194:795-823

Nielsen O, Egel R (1989) Mapping the double-strand breaks at the mating-type locus in fission yeast by genomic sequencing. EMBO J 8(1):269-276

Potten CS, Hume WJ, Reid P, Cairns J (1978) The segregation of DNA in epithelial stem cells. Cell 15(3):899-906 
Rosenberger RF, Kessel M (1968) Nonrandom sister chromatid segregation and nuclear migration in hyphae of Aspergillus nidulans. J Bacteriol 96:1208-1213

Sauer S, Klar AJS (2012) Left-right symmetry breaking in mice by left-right dynein may occur via a biased chromatid segregation mechanism, without directly involving the Nodal gene. Front Oncol 2(166):1-10

Schwarzacher HG, Schnedl W (1966) Position of labelled chromatids in diplochromosomes of endo-reduplicated cells after uptake of tritiated thymidine. Nature 209:107-108

Singh J, Klar AJS (1993) DNA polymerase-alpha is essential for mating-type switching in fission yeast. Nature 361(6409):271-273

Smith GH (2005) Label-retaining epithelial cells in mouse mammary gland divide asymmetrically and retain their template DNA strands. Development 132(4):681-687

Strathern JN, Klar AJS, Hicks JB, Abraham JA, Ivy JM, Nasmyth KA, McGill C (1982) Homothallic switching of yeast mating type cassettes is initiated by a double-stranded cut in the MAT locus. Cell 31(1):183-192

Styrkarsdottir U, Egel R, Nielsen O (1993) The smt-0 mutation which abolishes mating-type switching in fission yeast is a deletion. Curr Genet 23(2):184-186

Szostak JW, Orr-Weaver TL, Rothstein RJ, Stahl FW (1983) The double-strand-break repair model for recombination. Cell 33(1):25-35
Thon G, Friis T (1997) Epigenetic inheritance of transcriptional silencing and switching competence in fission yeast. Genetics 145(3):685-696

Tourte Y, Kuligowski-Andres J, Barbier-Ramond C (1980) Different behaviour of paternal and maternal genomes during embryogenesis in the fern, Marsilea. Eur J Cell Biol 21(1):28-36

Tran V, Lim C, Xie J, Chen X (2012) Asymmetric division of Drosophila male germline stem cell shows asymmetric histone distribution. Science 338:679-682

Vengrova S, Dalgaard JZ (2004) RNase-sensitive DNA modification(s) initiates $S$. pombe mating-type switching. Genes Dev 18(7):794-804

Walen KH (1965) Spatial relationships in the replication of chromosomal DNA. Genetics 51:915-929

Watson JD, Crick FH (1953) Molecular structure of nucleic acids; a structure for deoxyribose nucleic acid. Nature 171(4356):737-738

Yadlapalli S, Cheng J, Yamashita YM (2011) Drosophila male germline stem cells do not asymmetrically segregate chromosome strands. J Cell Sci 124:933-939

Yu C, Bonaduce MJ, Klar AJS (2013) Defining the epigenetic mechanism of asymmetric cell division of Schizosaccharomyces japonicus yeast. Genetics 193(1):85-94

Zhang Z, Shibahara K, Stillman B (2000) PCNA connects DNA replication to epigenetic inheritance in yeast. Nature 408(6809):221-225 